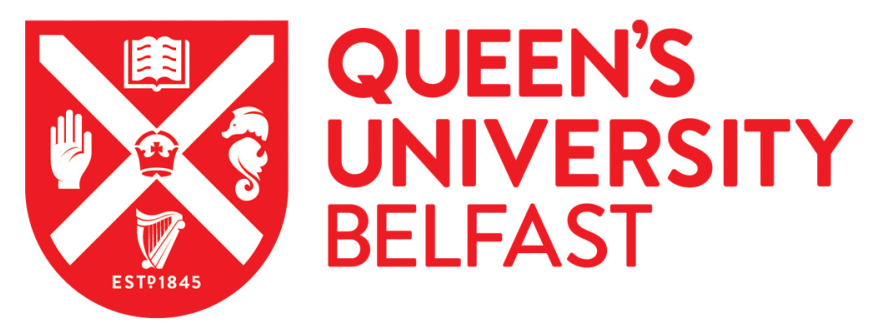

\title{
Experimental Study of Electrically Compact Retrodirective Monopole Antenna Arrays
}

Fusco, V., \& Malyuskin, O. (2017). Experimental Study of Electrically Compact Retrodirective Monopole Antenna Arrays. IEEE Transactions on Antennas and Propagation, 2339-2347.

https://doi.org/10.1109/TAP.2017.2684192

Published in:

IEEE Transactions on Antennas and Propagation

Document Version:

Peer reviewed version

Queen's University Belfast - Research Portal:

Link to publication record in Queen's University Belfast Research Portal

Publisher rights

(c) 2017 IEEE.

This work is made available online in accordance with the publisher's policies. Please refer to any applicable terms of use of the publisher.

\section{General rights}

Copyright for the publications made accessible via the Queen's University Belfast Research Portal is retained by the author(s) and / or other copyright owners and it is a condition of accessing these publications that users recognise and abide by the legal requirements associated with these rights.

Take down policy

The Research Portal is Queen's institutional repository that provides access to Queen's research output. Every effort has been made to ensure that content in the Research Portal does not infringe any person's rights, or applicable UK laws. If you discover content in the Research Portal that you believe breaches copyright or violates any law, please contact openaccess@qub.ac.uk. 


\title{
Experimental Study of Electrically Compact Retrodirective Monopole Antenna Arrays
}

\author{
Oleksandr Malyuskin, Vincent Fusco, Fellow, IEEE
}

\begin{abstract}
Auto-pointing and angular super-resolution properties of the radiation patterns generated by an electrically compact retrodirective monopole antenna array are demonstrated experimentally for the first time. The operation of electrically compact (element spacings less than one fifth of the radiation wavelength) retrodirective antenna arrays that were theoretically considered in our previous work are confirmed by measurement. Particularly it is shown that the Direction-of-Arrival (DoA) information carried by an incident electromagnetic wave can be encoded into the evanescent near field of an electrically small resonant antenna array with a spatial rate higher than the spatial oscillation rate of the incident field in free space. This observation is supported by the near field measurements demonstrating that the magnitude of the scattered (evanescent) field in the array environment can exceed the magnitude of the incident field in free space by at least $6 \mathrm{~dB}$. Retrodirective array antenna matching and the feasibility of a frequency-division full-duplex communication link based on the proposed antenna arrays are also discussed.
\end{abstract}

Index Terms - Antenna array, angular diffraction limit, monopole antennas, directive antennas, electromagnetic coupling, near field, radiation pattern, wireless communications

\section{INTRODUCTION}

$\mathrm{E}$ LECTRICALLY small antennas and antenna arrays have been extensively studied for several decades due to their theoretical and practical importance [1]-[6]. As a result of their small electrical size, these antennas and antenna arrays find many applications, including wireless communications, electromagnetic (EM) sensors, small aperture radars, compact microwave and mm-wave imaging systems [1], [6], [7]. Several interesting designs of electrically small antennas have been proposed recently. These include folded antenna geometries [1], [2], [6], [8], metamaterials [9]-[11], dielectric resonator antennas [1], [12] and biologically inspired resonantly loaded antennas [13], [14].

The design of electrically small antenna arrays with relatively high gain and practically useful bandwidth [3] represents a more challenging task than designing a single electrically small antenna, since the known rules for standard, half-wavelength spaced antenna arrays cannot be routinely applied due to complex near field EM coupling mechanisms [1], [3], [15].

An efficient approach to superdirective array realization [3] is based on selection of the electrically small resonant antenna

Manuscript received xx. 2016. The work is supported under the EPRSC grant EP/N020391/1 and the Leverhulme Trust UK research grant RPG-2013117. elements supporting large radiating currents that enable high unidirectional directivity of the order of 7-9dB even for simple 2-element antenna arrangements. It has been shown in [3], [15] that the spacings between the array elements cannot be made arbitrarily small due to accompanying significant radiation resistance drop, consequent antenna feeding mismatch and eventual antenna array efficiency reduction.

One of the essential properties of any antenna array is its ability to control the radiation pattern in receive and/or transmit mode. For the antenna arrays used in wireless communications an omnidirectional radiation pattern is commonly employed in the receive mode to ensure complete spatial coverage for signal reception [16]. At the same time, a narrow beam pattern radiated towards the communicator by an antenna array would add significant benefits to a communication system, particularly increased signal-to-noise ratio at the receiver for the same input power, lower bit-error rate (BER) reduced EIRP and EM pollution.

One class of electrically compact antenna arrays with electronically reconfigurable radiation patterns has been proposed in [17]. This antenna arrangement is based on a circular dipole or monopole antenna array topology with a central active element and a number of passive parasitic antennas with variable reactive loading [18], [19] (ESPAR antenna). ESPAR antennas allow electronic beam scanning with improved signal-to-noise ratio and reduced BER of the received signal, however ESPAR beam scanning requires considerable digital processing with regard to inputs related to the DoA and channel/propagation characteristics [18], [19].

In our previous companion paper [15] we proposed a fundamentally new class of electrically compact antenna terminals with omnidirectional radiation pattern in receive mode and auto-pointing superdirective radiation pattern in transmit mode, with auto-pointing requiring dedicated electronic circuitry performing EM signal phase conjugation, [20]- [22]. The EM phenomena enabling electrically compact retrodirective antenna array (RDA) operation are theoretically considered in full detail in [15]. Particularly, it has been demonstrated that the DoA information carried by an incident EM wave can be encoded into the array excitation vector in the receive mode with a spatial rate higher than the incident field spatial oscillation rate in free space. This can be understood by expanding the scattered field in the array environment into its plane wave spectrum where evanescent harmonics with fast

O. Malyuskin is with Ulster University, Shore Road, Newtownabbey Co. Antrim, BT37 0QB, UK. Email: o.malyuskin@ulster.ac.uk V. Fusco is with ECIT, Queen's University Belfast, Queen's Rd, BT39DT, UK. Email : v.fusco@ecit.qub.ac.uk 
spatial oscillation rate contribute dominantly into the Fresnel and quasistatic near field, [15], [23], [24]. This leads to much larger "effective" aperture of the dense array exceeding its geometrical area [25].

Next, phase conjugation of the receive excitation vector (amplitudes and phases of voltages across the antenna array terminals) and their re-application across the respective antenna terminals in transmit (retrodirective) mode leads to angular super-resolution spatial far-field patterns with auto-pointing properties. Angular super-resolution [25], [26] arises as a result of the "effective" aperture of the array being larger than its physical size which in turn requires high intensity of the evanescent field in the array environment that exceeds the magnitude of the incident field. This is possible when the antenna elements operate in EM resonance leading to high electric currents that generate large scattered field.

In this paper we experimentally study the EM performance of a 4-element retrodirective top-loaded resonance monopole array of compact electrical size $\sim \lambda / 4$, and inter-elementspacings $\lambda / 6, \lambda$ is the radiation wavelength. The retrodirection patterns measured in the azimuthal plane and the auto-pointing and angular super-resolution properties of these electrically compact RDA are demonstrated for the first time.

\section{Antenna ARray GeOMEtry And Measurement Setup}

\section{A. RDA Geometry}

A 4-element top-loaded monopole RDA, Fig. 1, is illuminated in the azimuthal plane by a vertically-polarized EM plane wave with monochromatic, $\exp (-i \omega t)$, frequency carrier.

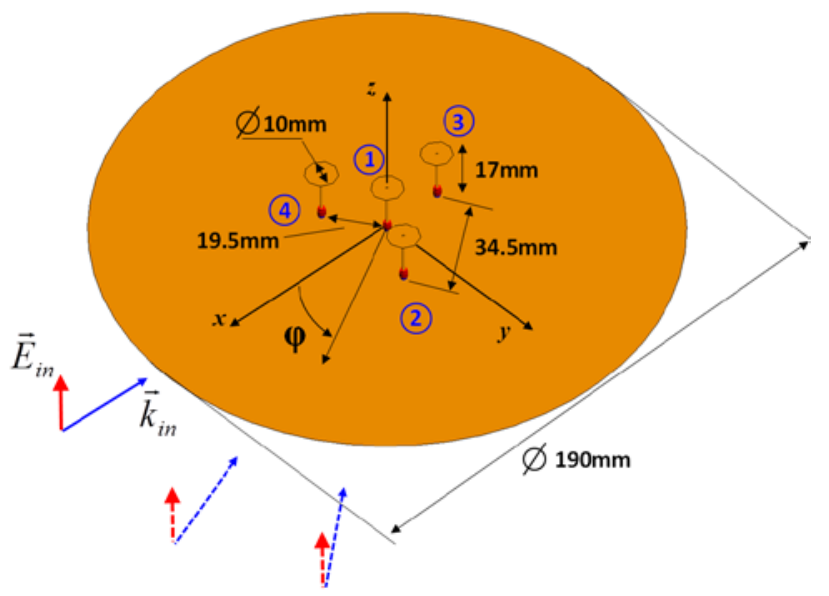

(a)

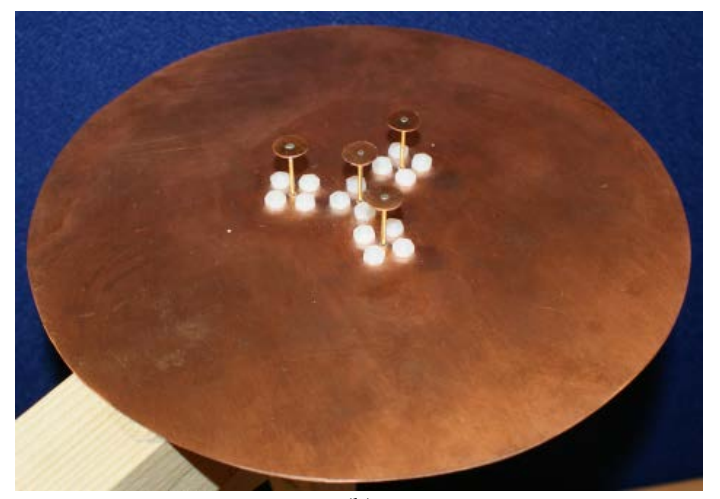

(b)

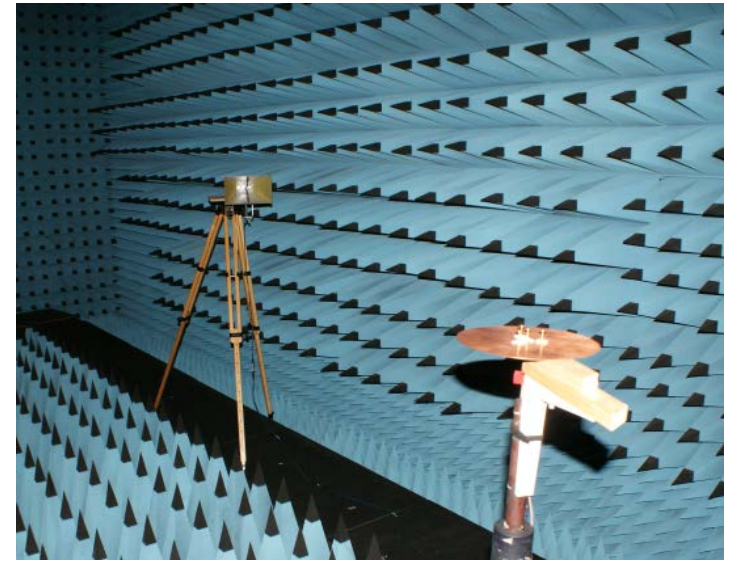

(c)

Fig.1. (a) Antenna geometry and FEKO simulation model; (b) fabricated prototype; (c) measurement setup in anechoic chamber. The distance between the horn antenna and RDA is $3.5 \mathrm{~m}$. In a) the antenna ports are labelled by the encircled numbers. In b) the monopole antennas are fed via 50 $\Omega$ SMA coaxial connectors with flanges attached to the circular ground plane by plastic bolts. The ground plane is mounted on a bracket and attached to a wooden turntable, inside the far-field anechoic range, c).

\section{B. Measurement Setup for the RDA in Receive Mode}

The measurement setup schematic diagram for the RDA in receive (RX) mode is shown in Fig. 2.

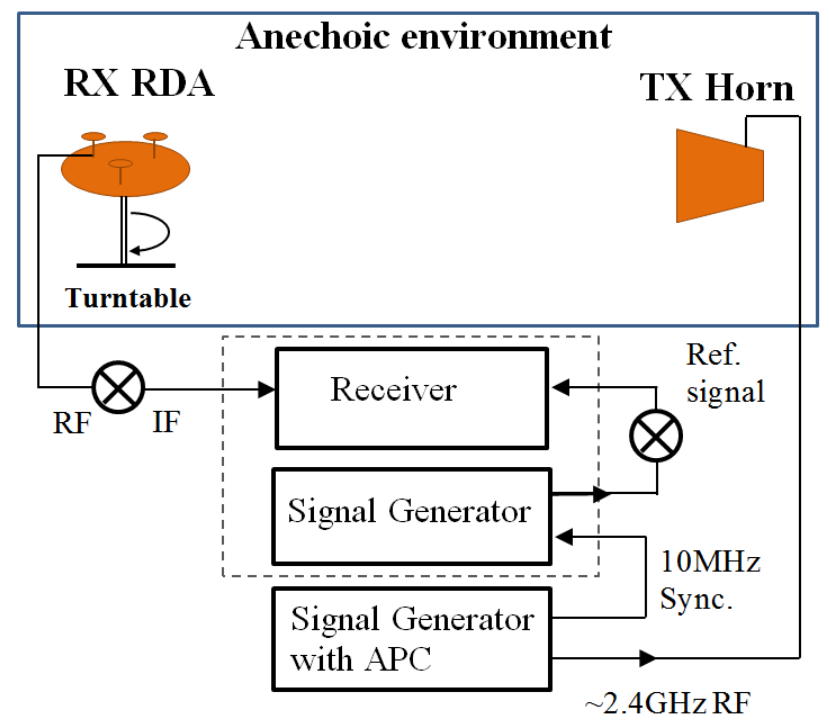

Fig.2. Measurement setup diagram for the compact RDA in receive mode.

In this arrangement the monopole RDA is illuminated by a transmitting (TX) vertically polarized horn antenna in the anechoic environment. The horn antenna is fed by a continuous wave signal at $\sim 2.4 \mathrm{GHz}$ generated by a signal generator Agilent E8257D with both amplitude and phase control (APC). This generator is synchronized at $10 \mathrm{MHz}$ with a signal generator/frequency synthesizer Hewlett Packard 8341B which forms a part of standard far-field anechoic chamber equipment (in dashed rectangle). The signal is received by each RDA port sequentially in the 360 degree range for the fixed TX horn 
position. All the ports except for the receiving one are left opened during the receive measurements. The RX signal is down converted to $1 \mathrm{GHz}$ and processed by the 1780 Scientific Atlanta amplitude and phase receiver thus RX amplitudes and phases across each RDA port are recorded for DoA in the 360degree range.

\section{Measurement Setup for the RDA in Retrodirective Mode}

In the RDA TX (retrodirective) mode the array excitation vector is formed using the amplitudes for the specific DoA and conjugated phases recorded at each array element, corresponding to this DoA. Phase conjugation can be realized by the analog or digital circuitry operators [22]. In this paper we generate the array excitation vector using a signal generator with amplitude and phase control since this allows us to measure the antenna array gain independent of particular phase conjugation circuitry realization. The excitation vector is applied to each port sequentially and the final far field radiation pattern obtained by linear superposition of the partial $\mathrm{RX}$ voltages at the horn antenna. The measurement setup diagram for the RDA TX mode is shown in Fig.3.

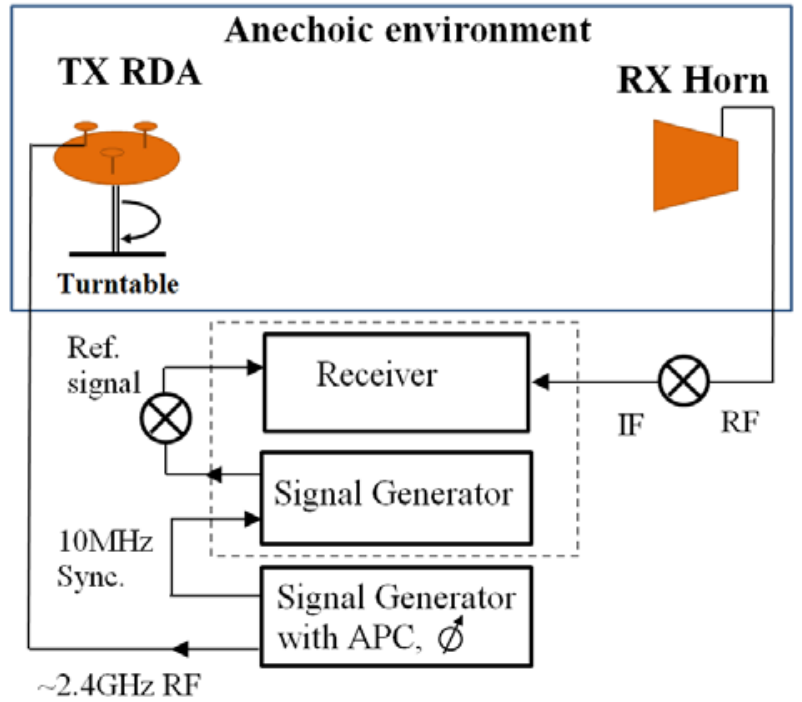

Fig.3. Measurement setup diagram for the RDA in TX/retrodirective mode.

\section{EXPERIMENTAL RESULTS}

\section{A. RDA Antenna Return Loss}

First we discuss RDA antenna matching. The monopole RDA in Fig. 1 has been designed to explore the effect of mutual near field coupling on the antennae feed port matching. It can be seen, Fig.1a), b) that there are three outer elements 2,3,4 that are exposed in a scattering field with magnitude smaller than the scattering field magnitude acting on central element 1 . It is well known [1], [3], [15] that larger reactive coupling between antennas (or, equivalently, near scattering field exposure) leads to lower radiation resistance and poorer antenna matching with respect to a $50 \Omega$ feeding port. This fact is illustrated by Fig. 4a) where the measured (using Agilent PNA network analyzer 8361C) and simulated return loss $\left|S_{n n}\right|$ is presented for antenna ports $n=1,2,3,4$ with reference port impedance $50 \Omega$. It can be seen that in the frequency band around $2.4-2.7 \mathrm{GHz}$ matching is acceptable for outer antenna elements and is very poor for the central antenna element which prevents its practical use.

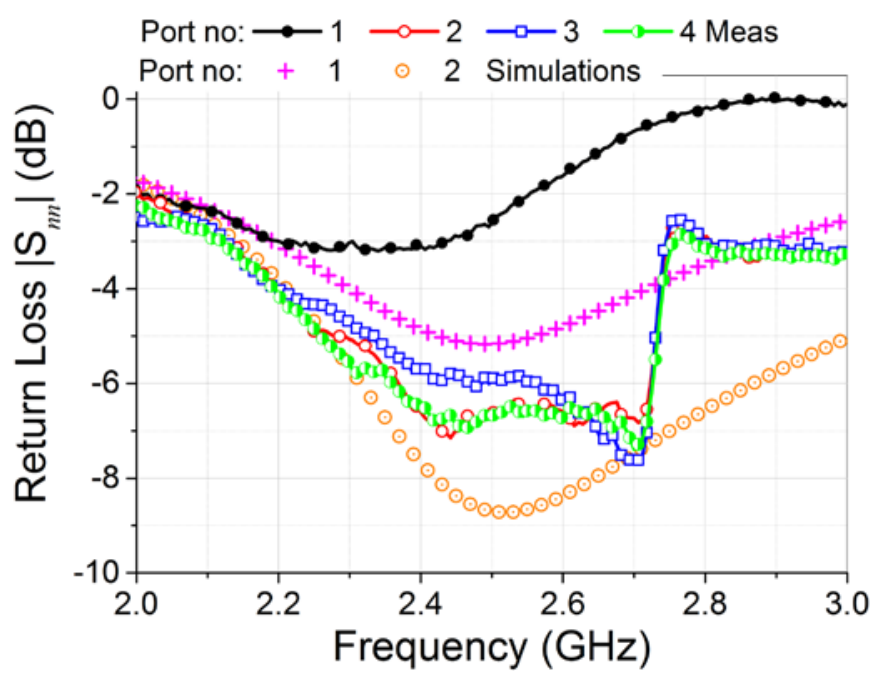

(a)

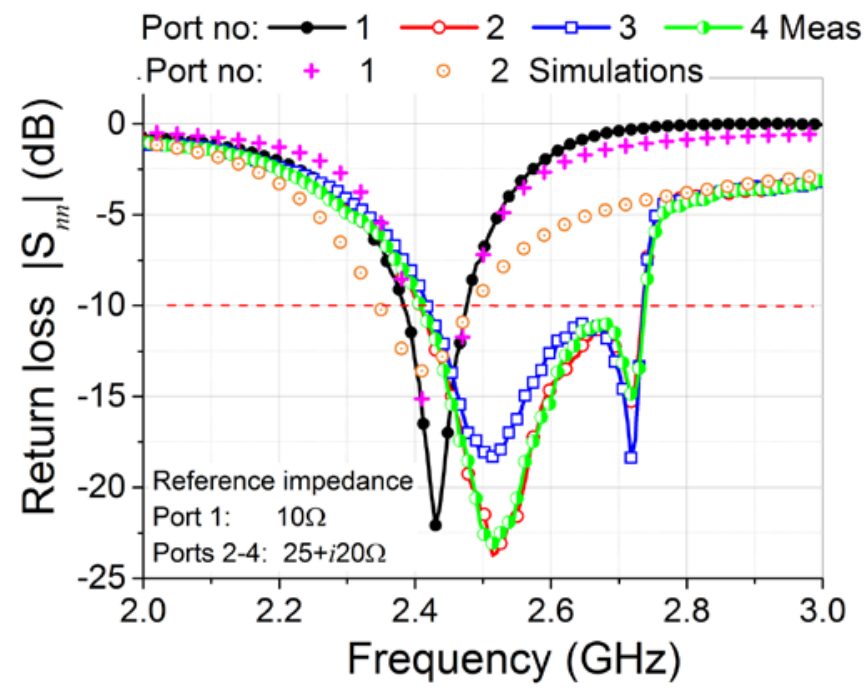

(b)

Fig.4. Simulated and measured return loss, $\left|\mathrm{S}_{n n}\right|$ for the RDA antenna ports $n=1,2,3,4$. (a) Reference port impedance $50 \Omega$ (b) Reference port impedance $10 \Omega$ for port 1 , impedance $25+i 20 \Omega$ for ports $2,3,4$ (measurements) and reference port impedance $25 \Omega$ for ports $2,3,4$ (simulations). FEKO [27] simulation data are the same for ports 2,3,4 and therefore only data for port 2 are shown. The difference between the simulated and measurement data is believed to be due to the point-like port model in FEKO not capturing fully the properties of the SMA feeding ports used in the experiment.

Consequently, a series of measurements has been performed to find out the optimal reference port impedance, Fig.4b). It can be seen that when the outer elements are fed through the ports with reference impedance $25+i 20 \Omega$ the antenna are wellmatched at $-10 \mathrm{~dB}$ in the $\sim 13 \%$ fractional bandwidth (2.4$2.73 \mathrm{GHz}$ ). At the same time the central element is matched only in $4 \%$ fractional bandwidth $(2.39-2.48 \mathrm{GHz})$. Also, the reference impedance for the central element is quite low, $10 \Omega$, and therefore matching for this element can suffer from losses within the antenna system. It should be noted that for the 
considered type of monopole RDA phase conjugated circuits can be printed on the back side of the ground plane and the required reference impedance can be obtained using lumped components, therefore $50 \Omega$ feeding is not a prerequisite.

\section{B.RDA in Receive Mode}

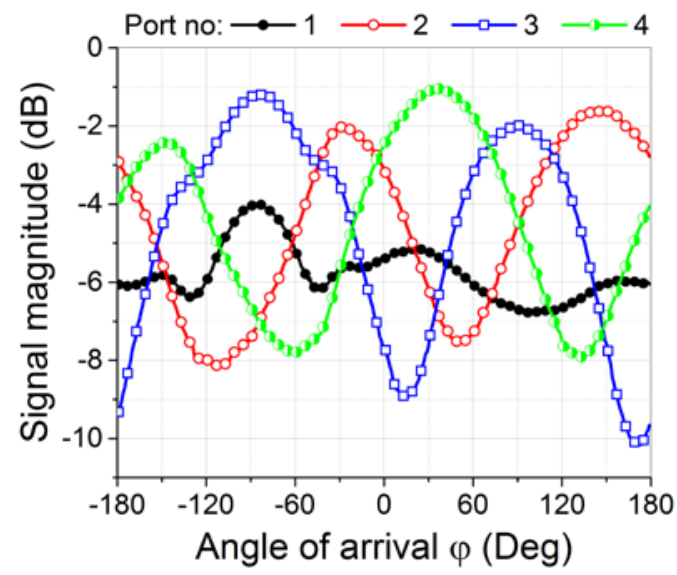

(a)

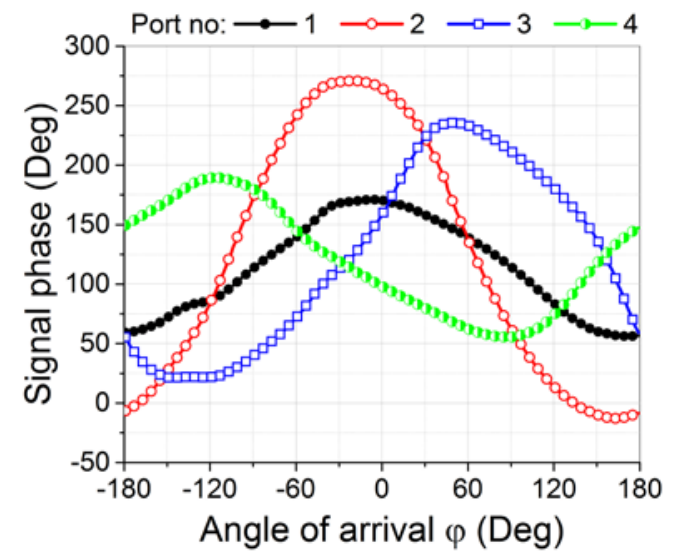

(b)

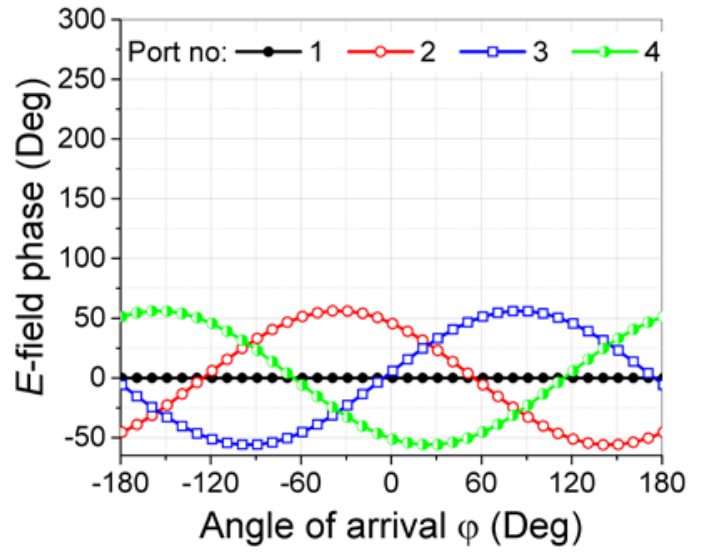

(c)

Fig.5.Measured RX signal magnitude (a) and phase (b) across the monopole RDA ports at $2.42 \mathrm{GHz}$. Simulated phase of the incident plane wave at the positions corresponding to monopole arm centers (with antenna elements removed). Asymmetry of the signal phase vs DoA characteristics between the ports 2,3,4, observed in Fig.5b) is believed to be caused by a small geometric difference between the antenna elements 2-4 and asymmetry of the ground plane due to fabrication tolerance.
Fig. 5 shows the measured received signal magnitude, Fig.5a) and phase, Fig.5b) across the RDA antenna ports at $2.42 \mathrm{GHz}$. Fig.5c) depicts the simulated phase of the incident plane wave in the positions corresponding to the monopole arm centers with the antennas removed. Graphs b), c) are plotted in the same scale for comparison. It can be seen that the RDA RX signal phases (and phase shifts between the ports) experience much larger variation (more than 100 degrees) with respect to the angle of arrivals than the respective phase of the incident field, [15]. Table I shows the measured voltage phase shifts between the respective antenna ports, Fig.5b) with the number in brackets standing for the respective phase shift acquired by the plane wave in free space at the locations corresponding to the respective ports positions, Fig.5c), for a number of DoAs.

\section{TABLE I. PHASE SHIFTS BETWEEN THE VOLTAGES ACROSS THE ANTENNA PORTS}

\begin{tabular}{lllll}
\hline \hline \multirow{2}{*}{$\varphi_{\text {DoA, }}{ }^{\circ}$} & \multicolumn{5}{c}{ Abs. value of the signal phase shift between the antenna } \\
& $1-2$ & $2-3$ & $3-4$ & $2-4$ \\
& & & & \\
& ports, ${ }^{\circ}$ & & \\
0 & $95(45)$ & $106(39)$ & $60(57)$ & $166(96)$ \\
60 & $2(6)$ & $95(56)$ & $171(96)$ & $75(39)$ \\
90 & $51(33)$ & $149(88)$ & $156(77)$ & $7(11)$ \\
120 & $70(51)$ & $167(96)$ & $107(39)$ & $60(57)$ \\
180 & $56(45)$ & $68(39)$ & $88(57)$ & $156(96)$ \\
\hline \hline
\end{tabular}

The larger phase shift variation, exceeding 100 degrees (corresponding to the largest separation $0.28 \lambda$ between the monopoles) can be explained [15] by the generation of large scattered field, $\vec{E}_{s c}(\vec{r})$ with magnitude exceeding the magnitude of the incident field in the dense antenna array environment. From [15], [23] the plane wave representation of the scattered field is

$$
\begin{aligned}
& \vec{E}_{s c}(\vec{r}) \sim \\
& \quad \sum_{n=1}^{N} \Phi_{n} \int_{-\infty}^{\infty} \overrightarrow{\mathrm{E}}_{s c}(\vec{k}) \exp \left[i \vec{\chi} \cdot\left(\vec{\rho}-\vec{\rho}_{n}\right)\right] \exp \left( \pm i k_{z} z\right) d \vec{\chi}
\end{aligned}
$$

where $\vec{r}=\vec{\rho}+z \vec{z}_{0}$ is the observation point radius vector, $\vec{\chi}$ is a spectral parameter (partial wavevector), $\vec{\rho}_{n}$ is the $n$-th antenna position in the azimuthal plane, $k_{z}^{2}+|\vec{\chi}|^{2}=k_{0}^{2}, k_{0}=2 \pi / \lambda$ is a free space wavenumber, and spectral amplitudes $\overrightarrow{\mathrm{E}}_{s c}(\vec{k})$ are given in [15].

In the near field the dominant role is played by the evanescent harmonics with wavenumbers $|\chi|>k_{0}, \chi=|\vec{\chi}|$, with wavenumbers exceeding the free space wave number $k_{0}$. These evanescent harmonics oscillate in the array plane with the spatial oscillation rate $\sim 2 \pi / \chi$ higher than the oscillation rate of the incident field (given by the wavelength $\lambda$ ) in free space. Therefore the DoA phase carried by the factor $\Phi_{n}=\exp \left(i \vec{k}_{i n} \cdot \vec{\rho}_{n}\right), \vec{k}_{i n}$ the incident wavevector is encoded into these evanescent components with higher spatial oscillation rate 
than is possible with the propagating waves in free space. This phenomenon manifests itself in significant phase shifts, $\sim \chi d$, $d$ is the antenna element spacings, between the received signal phases across the RDA antenna ports, Fig. 5b), as compared to the incident field phase variation in free space, Fig.5c), Table I. It should also be noted that another consequence of the resonance scattered field $\vec{E}_{s c}(\vec{r}) \gg \vec{E}_{\text {in }}(\vec{r})$ generated within the antenna array environment leads to the "effective" aperture [25] of the RDA being substantially larger than its geometric area resulting in angular super-resolution of its radiation patterns [15]. Direct experimental verification of the enhanced scattered field in the antenna array environment is provided in the next section.

\section{Near Field Measurement of the Scattered Field in the Antenna Array Environment}

The near field measurement setup of the RDA in receive mode is shown in Fig.6. The RDA is illuminated by a standard horn antenna located $1.4 \mathrm{~m}$ away in the near field anechoic chamber.
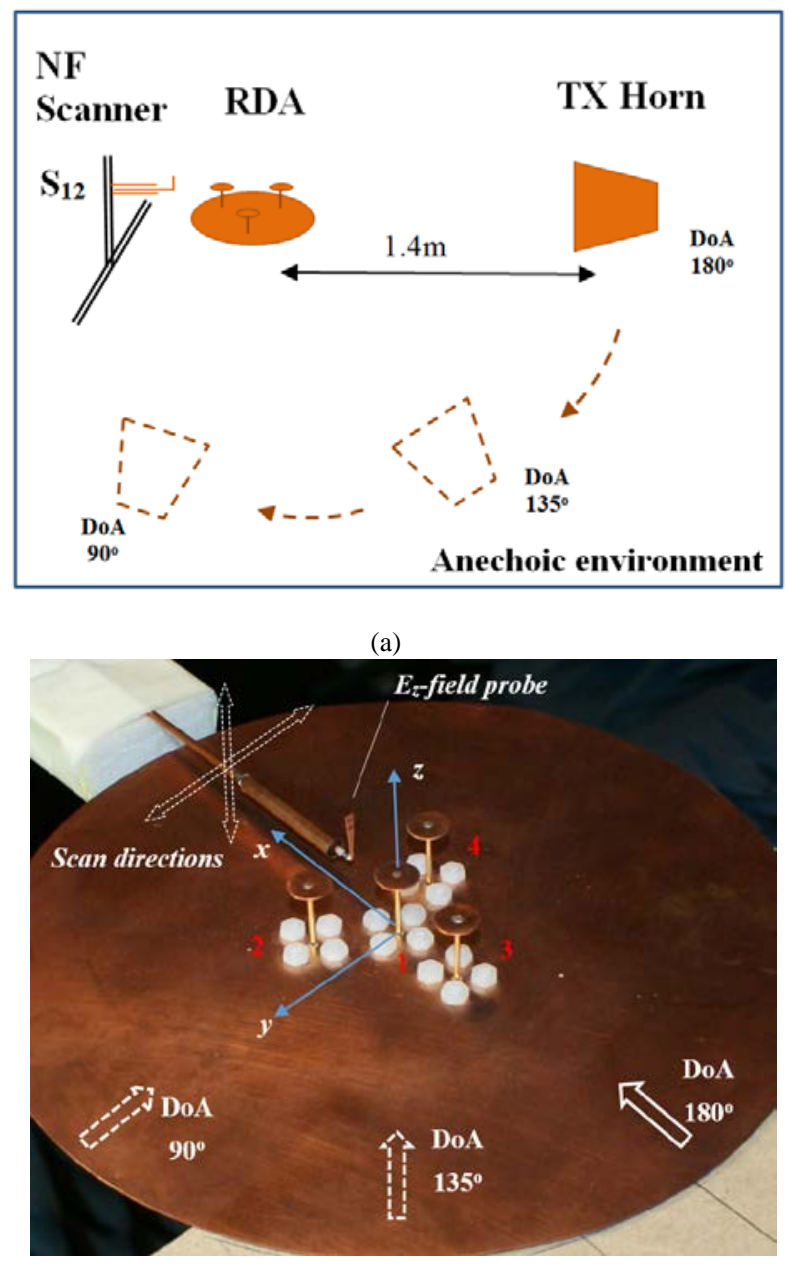

(b)

Fig.6. (a) Near field measurement setup geometry, (b) detailed view of the $E_{z^{-}}$ field probe in the vicinity of the 4-monopole array. The array is illuminated by a horn antenna at $\sim 2.4 \mathrm{GHz}$.

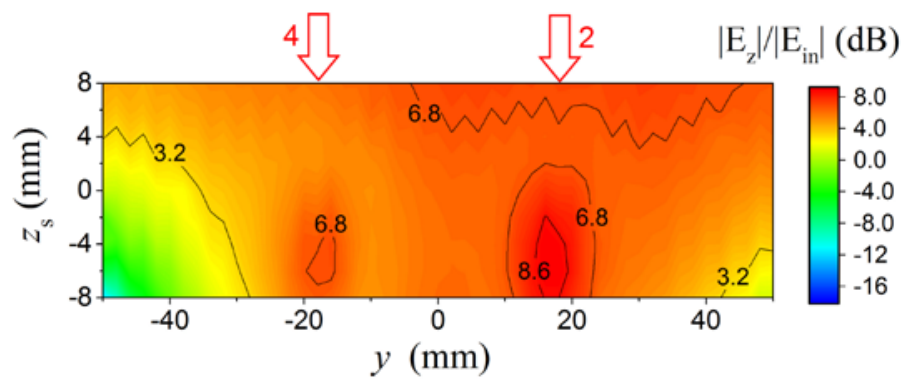

(a)

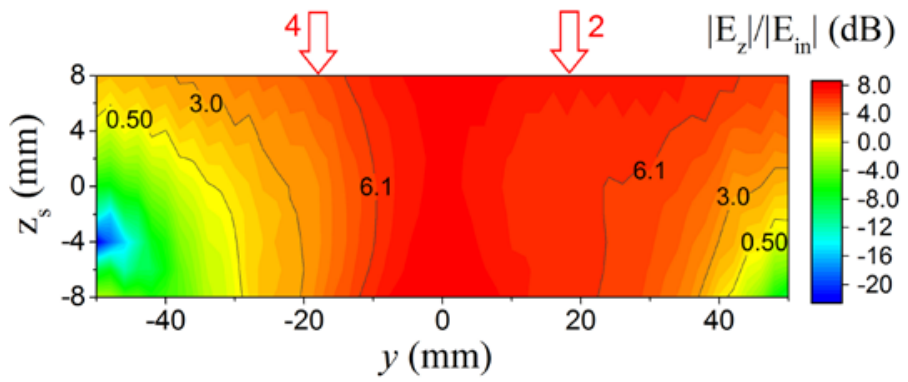

(b)

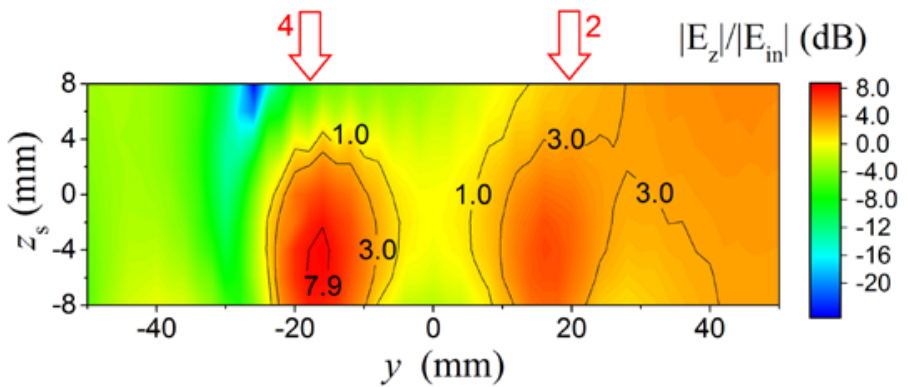

(c)

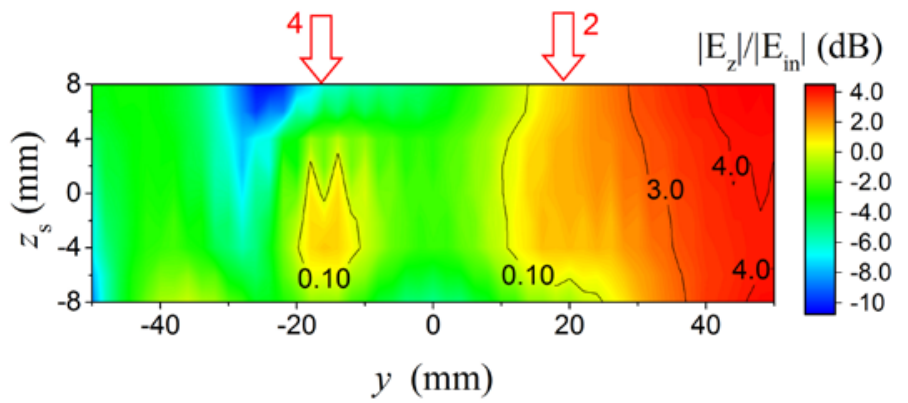

(d)

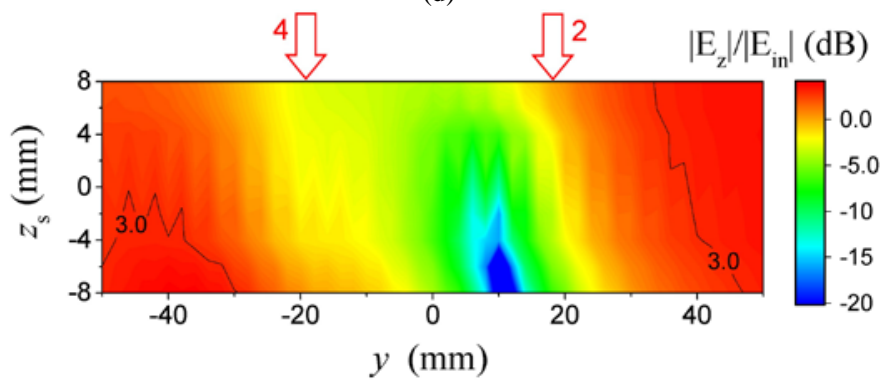

(e)

Fig.7. Near $E_{\mathrm{z}}$ field magnitude in the antenna array environment normalized by the magnitude of the incident field at $2.42 \mathrm{GHz}$. Normalized $E_{\mathrm{z}}$ magnitude in the yz plane at (a) $x=X_{o}=16 \mathrm{~mm}$, DoA $180^{\circ}$; (b) $X_{o}=20 \mathrm{~mm}$, DoA $180^{\circ}$; (c) $X_{o}$

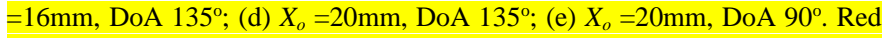
arrow markers on the graph tops show the positions of the monopoles 2 and 4 in the y range. 
The $E_{\mathrm{z}}$ field component is measured by an $8 \mathrm{~mm}$-long vertical probe, Fig.6b). First, z-component of the incident field $\vec{E}_{\text {in }}$ generated by the horn antenna is measured above the circular ground plane without the RDA.

Next, the vertical component (dominant in the vertical monopole array) of the total $E$ - field, arising as a sum $\vec{E}_{i n}+\vec{E}_{s c}$ of the incident and scattered fields is measured in the yz plane at the offset distance $x=X_{o}$ from the RDA center. Due to $120^{\circ}$ symmetry of the array geometry in the azimuthal range, it is sufficient to consider three DoAs, e.g. 90, 135 and 180 degrees, Fig.6.

The measured normalized vertical component of the total field, $\left|E_{z}\right| /\left|E_{\text {in }}\right|$ is shown in Fig.7. In these graphs the vertical axis $z_{s}$ origin is linked with the feed point of the $E_{z}$-field probe and displaced $10 \mathrm{~mm}$ up with respect to the global coordinate system $(x, y, z), z_{s}=z+10 \mathrm{~mm}$. It can be seen that for the DoA $180^{\circ}$, the magnitude of the total field in the array environment exceeds the incident field magnitude by at least $8 \mathrm{~dB}$ which in turns demonstrates substantial enhancement of the scattered field within the RDA environment. As the angle of arrival changes from $180^{\circ}$ to $135^{\circ}$ and subsequently $90^{\circ}$, the total field magnitude in the yz plane (at Xo offset distance) is reduced due to the near field interference mechanisms, not studied here in detail for the brevity's sake.

Since the $E_{\mathrm{z}}$-field probe, Fig. 6b), collects both the propagating and evanescent parts of the EM spectrum, further insight into the spatial spectral content of the EM field in the antenna array environment is required using an analytical approach presented in the following section.

\section{Propagating and Evanescent Field in the Antenna Array Environment}

This section analyses the EM spatial spectrum content in the RDA environment. First, let us represent the spatial Green's function $G\left(\vec{r}, \vec{r}^{\prime}\right)$ of a point source as a sum of propagating and evanescent parts,

$$
G\left(\vec{r}, \vec{r}^{\prime}\right)=\frac{\exp \left(i k_{0} R\right)}{4 \pi R}=G_{\text {prop }}\left(\vec{r}, \vec{r}^{\prime}\right)+G_{e v}\left(\vec{r}, \vec{r}^{\prime}\right)
$$

where

$$
\begin{aligned}
& G_{\text {prop }}\left(\vec{r}, \vec{r}^{\prime}\right)=\frac{i}{4 \pi} \int_{0}^{k_{0}} \frac{\exp \left(i \sqrt{k_{0}^{2}-\chi^{2}}|\tilde{z}|\right)}{\sqrt{k_{0}^{2}-\chi^{2}}} J_{0}(\chi \tilde{\rho}) \chi d \chi, \\
& G_{e v}\left(\vec{r}, \vec{r}^{\prime}\right)=\frac{1}{4 \pi} \int_{k_{0}}^{\infty} \frac{\exp \left(-\sqrt{\chi^{2}-k_{0}^{2}}|\tilde{z}|\right)}{\sqrt{\chi^{2}-k_{0}^{2}}} J_{0}(\chi \tilde{\rho}) \chi d \chi .
\end{aligned}
$$

The propagating and evanescent parts of the point source Green's function in the form (3), (4) can be obtained using a standard procedure detailed in, e.g. [28]. In (3), (4) $\tilde{\rho}=\rho-\rho^{\prime}$ and $\tilde{z}=z-z^{\prime}$ and $J_{0}$ is Bessel's function of the zero order, first kind. In (2) $R=\sqrt{\tilde{\rho}^{2}+\tilde{z}^{2}}$. Integral (3) is regularly convergent for moderate values of $\tilde{\rho}$ of few wavelengths. At the same time the integral (4) is poorly convergent superoscillatory integral when $|\tilde{z}| \approx 0$. Integral representation (4) can be regularized by subtracting and adding the asymptotic term $(1 / 4 \pi) \int_{k_{0}}^{\infty} J_{0}(\chi \tilde{\rho}) \chi d \chi$, which results in the following regularized expression for the evanescent part of the Green's function

$$
\begin{aligned}
& G_{e v}\left(\vec{r}, \vec{r}^{\prime}\right)= \\
& \frac{1}{4 \pi} \int_{k_{0}}^{\infty}\left[\frac{\exp \left(-\sqrt{\chi^{2}-k_{0}^{2}}|\tilde{z}|\right)}{\sqrt{\chi^{2}-k_{0}^{2}}} \chi-1\right] J_{0}(\chi \tilde{\rho}) d \chi+G_{s}\left(\rho, \rho^{\prime}\right)
\end{aligned}
$$

where the term $G_{s}\left(\rho, \rho^{\prime}\right)$ is given by the following expression

$$
\begin{aligned}
& G_{s}\left(\rho, \rho^{\prime}\right)= \\
& \frac{1}{4 \pi \tilde{\rho}}\left(1-k_{0} \tilde{\rho}+\frac{\pi k_{0} \tilde{\rho}}{2} \cdot\left(J_{0}\left(k_{0} \tilde{\rho}\right) \mathbf{H}_{1}\left(k_{0} \tilde{\rho}\right)-J_{1}\left(k_{0} \tilde{\rho}\right) \mathbf{H}_{0}\left(k_{0} \tilde{\rho}\right)\right)\right)
\end{aligned}
$$

In (6) $\mathbf{H}_{0}, \mathbf{H}_{1}$ are Struve functions [29], [30] easily computed in terms of the polynomial series and $J_{1}$ is Bessel's function of the first order, first kind. It is interesting to note that the first-order term in series (6) is a point-source quasi-static Green's function in the azimuthal plane. Integral (5) possesses good convergence properties for arbitrary $\tilde{Z}$ and can be efficiently calculated using asymptotic techniques [31].

Next, to evaluate the scattered field inside the monopole RDA environment one can use the expression [32] for the vertical, $z$-component of the EM field generated by a monopole antenna,

$E_{z}\left(\vec{r}, \vec{r}^{\prime}\right)=$

$\frac{i Z_{0}}{4 \pi k_{0}}\left(\int_{0}^{h} G\left(\vec{r}, \vec{r}^{\prime}\right) \cdot\left(\frac{\partial^{2}}{\partial z^{\prime 2}}+k_{0}^{2}\right) I\left(z^{\prime}\right) d z^{\prime}-\left.G\left(\vec{r}, \vec{r}^{\prime}\right) \frac{\partial I\left(z^{\prime}\right)}{\partial z^{\prime}}\right|_{0} ^{h}\right)$

In (7) $Z_{0}$ is a free space impedance, and $I(z)$ is the current distribution along the vertical wire of the monopole antenna. Using expression (2)- (6) in (7) one can easily calculate the propagating and evanescent $E_{z}$-field spatial spectrum content in the EM field generated in the RDA environment. 


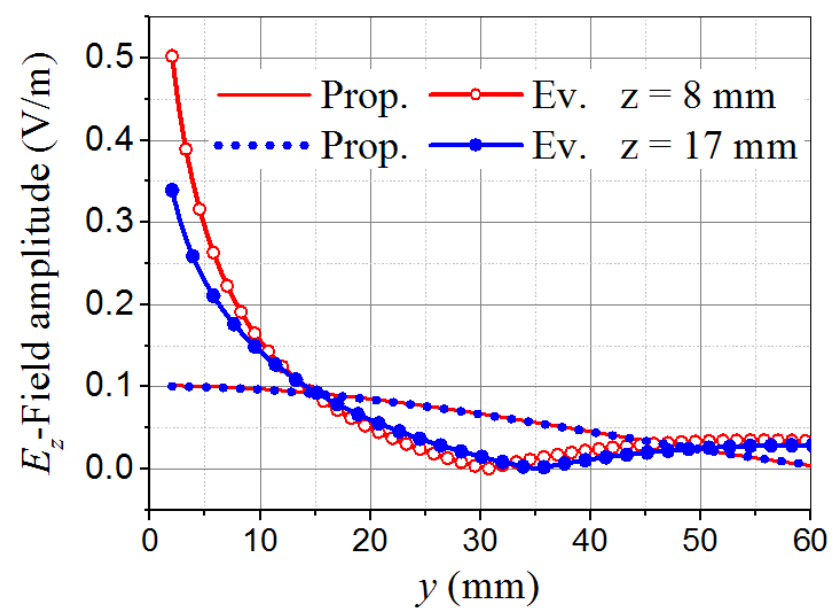

(a)

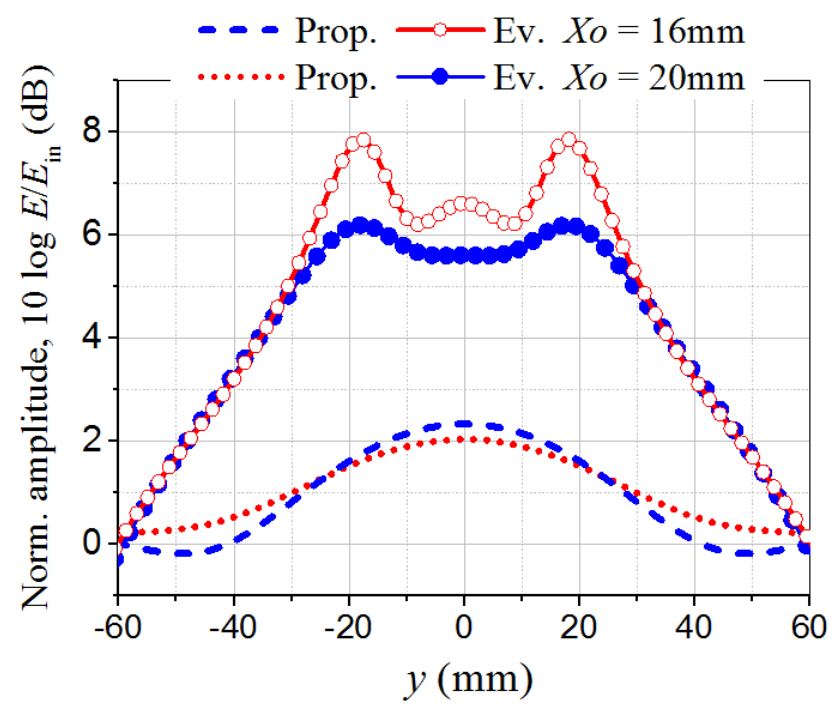

(b)

Fig.8. a) Ez-field magnitude of a single base-fed (with $1 \mathrm{mV}$ base voltage source) monopole antenna in the horizontal plane at $\mathrm{z}=8 \mathrm{~mm}$ and $\mathrm{z}=17 \mathrm{~mm}$. b) Normalized Ez-field magnitude in the yz plane scattered by the 4-element RDA with dimensions specified in Fig.1a) for DoA $180^{\circ}$, at two stand-off distances, $X o=16 \mathrm{~mm}$ and $X o=20 \mathrm{~mm}$, cf. Fig.6b).

Fig. 8 shows calculated (7) data of the evanescent and propagating $E_{z}$-field magnitude generated by a single monopole antenna of $17 \mathrm{~mm}$ height and $0.5 \mathrm{~mm}$ radius, base-fed with a voltage source of $1 \mathrm{mV}$. The electric current distribution $I(z)$ along the monopole vertical wire is derived from a full-wave FEKO simulation model [27]. It can be seen, Fig.8a), that the evanescent $E_{z}$-field dominates over the propagating counterpart in the vicinity of the antenna element (at distances less than $10 \mathrm{~mm}$ ). In the RDA environment both evanescent and propagating fields experience near field interference which can significantly change the field distribution pattern. Consider for example the situation shown in Fig.6b), the RDA in RX mode for DoA $180^{\circ}$. In this case, the field in the yz plane is substantially enhanced with respect to the incident field, Fig.7a),b). The calculated evanescent and propagating $E_{\mathrm{z}}$-field magnitude for this case is shown in Fig.8b). The array excitation vectors and current distributions are obtained using a full-wave FEKO RDA model. Firstly, Fig.8b) demonstrates very good agreement with experimental data in Fig.7a), b). Secondly Fig.8b) shows that near field interference leads to a very high magnitude of the evanescent field in the entire antenna array environment (in the range $-30 \mathrm{~mm}<\mathrm{y}<30 \mathrm{~mm}$ ) exceeding the propagating part of the $E_{z}$-field by at least $4-6 \mathrm{~dB}$.

\section{E. RDA in Transmit Mode}

In the retrodirective mode the phase-conjugated array excitation vector on receive is re-applied to the respective RDA antenna terminals [15]. Since only one signal generator was available for the amplitude and phase control, the RDA ports were excited sequentially, and the resulting far-field spatial patterns obtained using linear superposition of the signals corresponding to each of the individual port excitations.

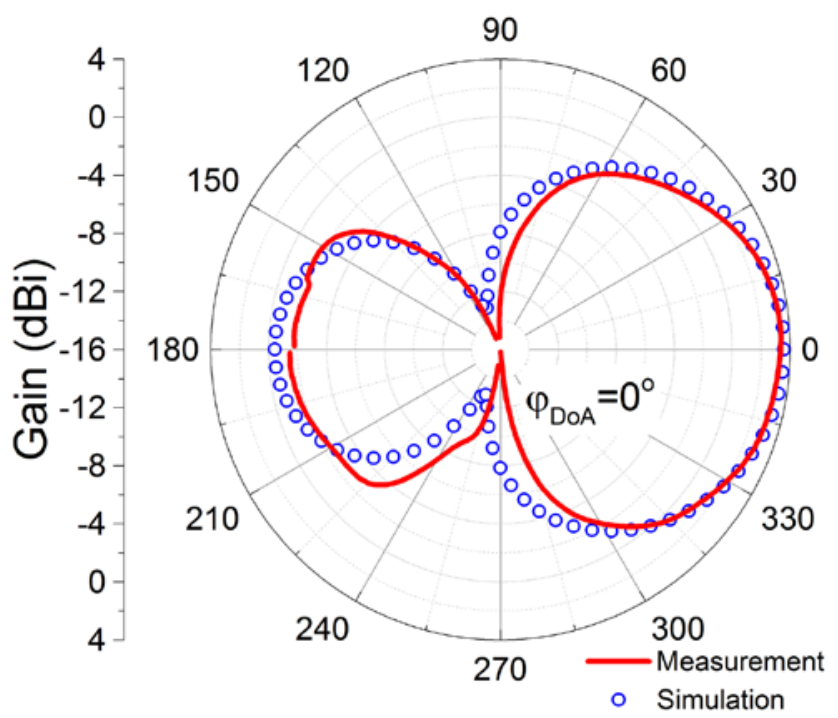

(a)

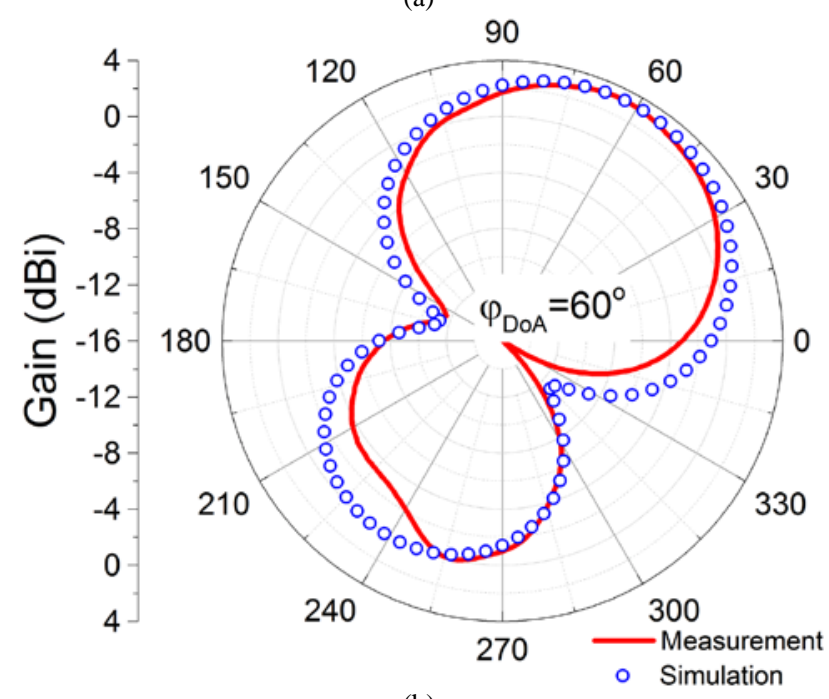

(b) 


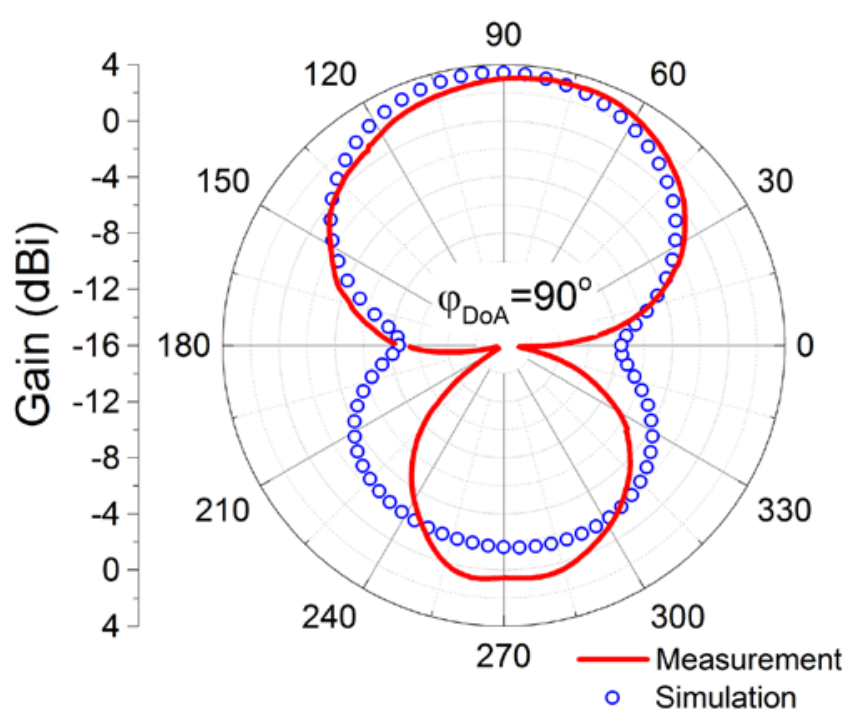

(c)

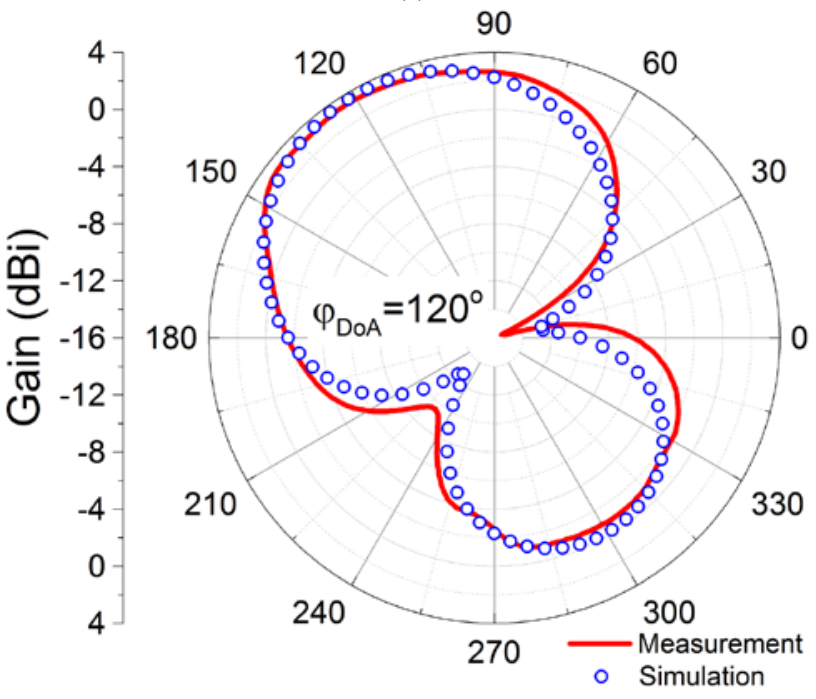

(d)

Fig.9. Measured (solid lines) and simulated (hollow dots) radiation patterns for DoA 0, 60, 90 and 120 degrees.

\section{TABLE II. ARRAY EXCITATION VECTORS IN TX MODE}

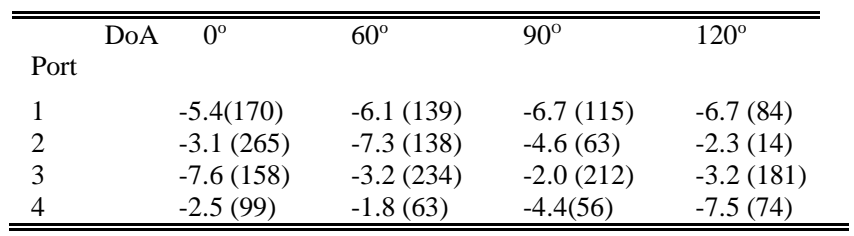

In order to calculate relative gain, a reference dipole was used so that gain was calculated as a ratio of the signal power received by the horn antenna from the RDA array to the power received by the horn from a reference dipole, for the same input power. Since the standard definition of gain does not include antenna mismatch loss [3], the return loss was taken at $-15 \mathrm{~dB}$, for $50 \Omega$ feeding.

The "realized" gain including mismatch losses is smaller by approximately $30 \%$. It should be noted that for the reference port impedance matching as in Fig.4b) the "realized" gain is the same as the relative gain presented in Fig.9. The retrodirective radiation patterns were measured at $2.42 \mathrm{GHz}$ in the DoA range $0-120^{\circ}$ with $30^{\circ}$ step, the results are shown in Fig.9. Table II shows the array excitation vectors across the RDA ports in the form "amplitude(-phase)" with amplitude in $\mathrm{dB}$ and phase in degrees. It should be noted that the RDA retrodirects in the full $360^{\circ}$ azimuthal range [15], demonstration in $0-120^{\circ}$ span is sufficient due to the monopole array azimuthal symmetry. In Fig.9, full-wave FEKO simulations are also presented for comparison.

Figs.9a)-d) show that the radiation pattern angular bandwidth $\Delta \theta_{-3 \mathrm{~dB}}$ at $-3 \mathrm{~dB}$ level is around 90 degrees regardless the DoA angle. The resolution-limited radiating aperture [25] corresponding to the RDA size $L \sim \lambda / 4$ would generate a pattern with beamwidth $\Delta \theta_{-3 \mathrm{~dB}} \approx \lambda / L$ (Rad) of 4 radians (229 degrees).

F. Frequency-division full-duplex communication link feasibility

Let us consider now the feasibility of a frequency-division full-duplex communication link based on the electrically compact RDAs. In this scenario the communicator sends a downlink signal towards the RDA at the carrier frequency $f_{1}$ and receives the uplink signal at the carrier frequency $f_{2}$. From the practical point of view [22] it is interesting to establish if the retrodirective properties of the RDA are preserved if the array excitation vector obtained at the frequency $f_{1}$ is applied to generate the retrodirected signal at carrier frequency $f_{2}$. The measurement results carried out for DoA 0 degrees are shown in Fig.10. It can be seen that when the carrier frequency deviates within $1 \%$ the retrodirective properties of the compact monopole RDA are fairly well preserved. However, carrier frequency deviation of more than $1 \%$ leads, in general, to significant retrodirective pattern pointing error. Therefore if a frequency-division full-duplex link is required, a carrier signal at downlink frequency $f_{1}$ has to be accompanied with a pilot signal at the uplink frequency $f_{2}$ to generate an uplink array excitation vector.

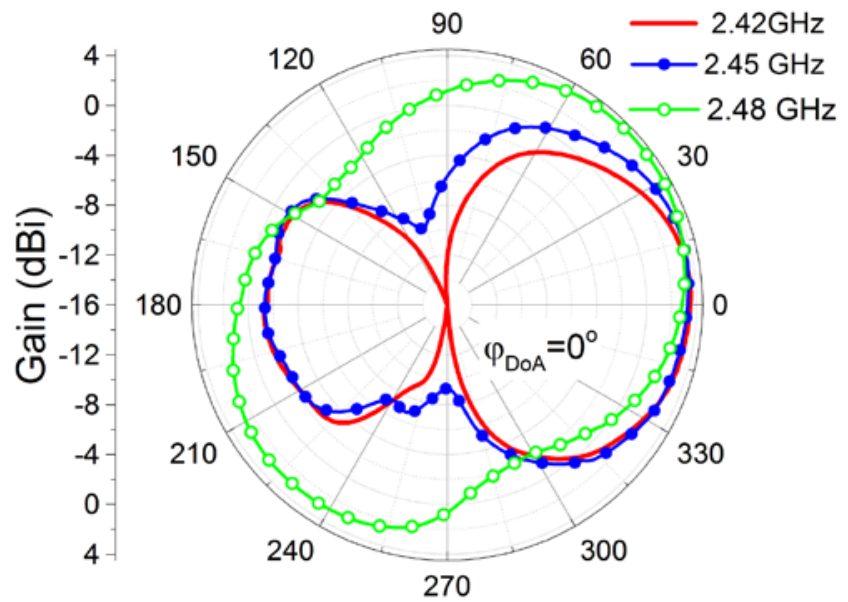

Fig.10. Measured retrodirective radiation patterns for the situation when the monopole RDA excitation vector at $2.42 \mathrm{GHz}$ is used to generate the retrodirective radiation patterns at close frequencies $2.45 \mathrm{GHz}$ and $2.48 \mathrm{GHz}$. 


\section{CONCLUSIONS}

Angular super-resolution and auto-pointing properties of the radiation patterns generated by an electrically compact retrodirective monopole antenna array have been shown experimentally. Particularly it was demonstrated that the angular beamwidth at $-3 \mathrm{~dB}$ of a four-element array with a quarter-wavelength characteristic spatial size is 90 degrees for an arbitrary DoA. In contrast, the resolution-limited angular bandwidth of the quarter-wavelength aperture radiation pattern is around 230 degrees. Thus arrays of the proposed type enable physical super-resolution based on DoA information encoding into the highly oscillatory evanescent harmonics generated within the antenna array environment. Substantial evanescent near field enhancement within the RDA has been also shown experimentally and analytically for the first time.

It should be noted that the mechanism of super-resolution presented in this paper is essentially different from the classical super-resolution phenomenon based on the super-oscillatory behavior of the antenna element currents and fields [33]. This in turn means that the proposed antenna arrays are free from the drawbacks pertinent to the classical super-oscillatory arrays [15], [34], namely extremely narrow frequency bandwidth of operation, high sensitivity to geometrical tolerances, etc.

Moreover, it has been shown that for antenna elements with $\lambda / 6$ spacings, a $13 \%$ fractional bandwidth of return loss at $-10 \mathrm{~dB}$ level can be readily achieved by tuning the reference impedance within practical limits. A very attractive feature of the proposed compact arrays in their ability to retrodirect the signal automatically without any digital signal processing or DoA estimation. No compensation of antenna coupling effects is also required.

It is believed that the proposed antenna terminals could find applications in energy-efficient wireless communications, and EM systems where space is a premium.

\section{ACKNOWLEDGEMENT}

The authors are thankful to Mr. Kieran Rainey for fabrication of the monopole RDA prototype and assistance with the measurements. Also the authors thank anonymous reviewers for helpful comments and suggestions that allowed us to improve original manuscript.

\section{REFERENCES}

[1] R.C. Hansen, R. E. Collin, Small Antenna Handbook, John Wiley \& Sons, Inc. 2011.

[2] K. Fujimoto, A. Henderson, K. Hirasawa, and J. R. James, Small Antennas, Wiley, 1987.

[3] A. D. Yaghjian, T. H. O’Donnell, E. E. Altshuler, and S. R. Best, "Electrically small supergain end-fire arrays", Radio Science, vol. 43, RS3002, pp1-13, 2008.

[4] Altshuler, E. E., T. H. O’Donnell, A. D. Yaghjian, and S. R. Best, “A monopole superdirective array”, IEEE Trans. Antennas Propag., vol. 53, no. 8, pp. 2653-2661, Aug. 2005.

[5] R.W.P. King, "Supergain Antennas and the Yagi and circular arrays", IEEE Trans Antennas Propagat., vol.37, no.2, pp. 178-186, Feb.1989.

[6] M. J. Slater, C.D. Schmitz, M.D. Anderson, et al., "Demonstration of an Electrically Small Antenna Array for UHF Direction-of-Arrival Estimation”, IEEE Trans Antennas Propagat., vol.61, no.3, pp. 13711377, Mar 2013.
[7] H.-T. Liu, S. Gao, and T.-H. Loh, "Electrically Small and Low Cost Smart Antenna for Wireless Communication”, IEEE Trans. Antennas Propagat., vol.60, no.3, pp. 1540-1549, March 2012.

[8] S. Best, "The performance properties of electrically small resonant multiple-arm folded wire antennas”, IEEE Antennas Propag. Mag., vol. 47, pp. 13-27, 2005.

[9] R. W. Ziolkowski, P. Jin, and C.-C. Lin, "Metamaterial-Inspired Engineering of Antennas”, Proc. IEEE, vol. 99, pp. 1720-1731, 2011.

[10] A. D. Yaghjian, "Increasing the supergain of electrically small antennas using metamaterials," Proc. 3rd Eur. Conf. Antennas Propag. (EuCAP’09), 2009, pp. 858-860.

[11] K. Buell, H. Mosallaei and K. Sarabandi, "Metamaterial Insulator Enabled Superdirective Array”, IEEE Trans. Antennas Propagat., vol. 55, no.4, pp. 1074-1085, 2007.

[12] Y.-M. Pan, K. W. Leung, and Kai Lu,“Compact Quasi-Isotropic Dielectric Resonator Antenna With Small Ground Plane”, IEEE Trans. Antennas Propagat., vol.62, no.2, pp. 577-585, Feb. 2014.

[13] N. Behdad, M.A. Al-Joumayly and M. Li, "Biologically Inspired Electrically Small Antenna Arrays with Enhanced Directional Sensitivity”, IEEE Trans. Antennas Propagat. Letters, vol.10, pp. 361-364, 2011.

[14] N. Behdad, M.A. Al-Joumayly and M. Li, "Biologically-Inspired Antenna Arrays Based on the Hearing Mechanism of the Parasitoid Fly Ormia Ochracea”, IEEE Int. Symp. Antennas. Propagat. (APSURSI 2011), pp. 1526-1529, 2011.

[15] O. Malyuskin, V. Fusco, "Ultracompact Retrodirective Antenna Arrays with Superdirective Radiation Patterns”, IEEE Trans. Antennas Propagat., vol.64, no.7, pp. 2923 - 2935, July 2016.

[16] S. Saunders, A. Aragón-Zavala, Antennas and Propagation for Wireless Communication Systems, Wiley 2007.

[17] C. Sun, A. Hirata, T. Ohira, N. Karmakar, "Fast Beamforming of Electronically Steerable Parasitic Array Radiator Antennas: Theory and Experiment”, IEEE Trans. Antennas Propagat., vol.57, no.7, pp. 18191832, July 2004.

[18] H.-T. Liu, S. Gao, and T.-H. Loh, "Compact MIMO Antenna with Frequency Reconfigurability and Adaptive Radiation Patterns", IEEE Trans. Antennas Propagat. Lett., vol. 12, pp. 269-272, 2013.

[19] Y. Zhou, R. S. Adve, Sean Victor Hum, "Design and Evaluation of Pattern Reconfigurable Antennas for MIMO Applications”, IEEE Trans. Antennas Propagat., vol.62, no.3, pp. 1084-1092, March 2014.

[20] V. Fusco, O. Malyuskin, N. Buchanan, "Active phase conjugating lens with sub-wavelength resolution capability", IEEE Trans. Antennas Propagat., vol.58, no.3, pp. 798-808, 2010.

[21] O. Malyuskin, V. Fusco, A. Schuchinsky, "Microwave phase conjugation using nonlinearly loaded wire arrays”, IEEE Trans. Antennas Propagat., vol.54, no.1, pp. 192-203, 2006.

[22] V. Fusco, N. Buchanan, "Developments in retrodirective array technology”, IET Microw. Antennas Propagat., vol. 7, no. 2, pp. 131-140, 2013.

[23] T.B. Hansen, A.D. Yaghjian, Plane-Wave Theory of Time-Domain Fields: Near-Field Scanning Applications, Wiley IEEE, 1999.

[24] O. Malyuskin, V. Fusco, "Near field focusing using phase conjugating impedance loaded wire lens”, IEEE Trans. Antennas Propagat., vol. 58, no.9, pp. 2884-2893, 2010.

[25] E.V. Jull, Aperture Antennas and Diffraction Theory, Peter Peregrinus, 1981.

[26] R.C. Hansen, "Fundamental limitations in antennas", Proceedings IEEE, vol. 69, no.2, pp. 170-182, Feb. 1981.

[27] Electromagnetic solver FEKO, www.feko.info

[28] L. Brekhovskikh, Waves in Layered Media, 2nd Edition, Academic Press, 1980, Chapter 4.

[29] I. S. Gradshteyn and I. M. Ryzhik, Table of Integrals, Series, and Products. New York: Academic, 1980.

[30] M. Abramowitz and I. A. Stegun, Handbook of Mathematical Functions. New York: Dover, 1972, Chapter 12.

[31] O. Malyuskin; V. Fusco; A. Schuchinsky, "Convergence acceleration of the doubly periodic Green's function for the analysis of thin wire arrays", IET Microwaves, Antennas \& Propagation, vol. 2, no. 5, pp. 410-417, 2008

[32] C. Harrison, C. Taylor, E. Aronson, M. Houston, "An Accurate Representation of the Complete Electromagnetic Field in the Vicinity of a Base-Driven Cylindrical Monopole", IEEE Trans. Electromagn. Compatibility, vol. 12, no. 4, pp.164-173, Nov. 1970. 
[33] E.T.F. Rogers, J. Lindberg, T. Roy, et al, “A super-oscillatory lens optical microscope for subwavelength imaging”, Nature Materials Lett. Vol.11, pp. 432-435, May 2012.

[34] N. Yaru, "A note on super-gain antenna arrays," Proc. IRE, vol 39, pp. 1081-1085, Sep. 1951. 